\title{
Surtos de infecção por Piscinoodinium pillulare e Henneguya spp. em pacus (Piaractus mesopotamicus) criados intensivamente no Sudoeste de Goiás ${ }^{1}$
}

\author{
Fabiano J.F. de Sant'Ana ${ }^{2 *}$, Sílvio L. de Oliveira ${ }^{3}$, Rogério E. Rabelo ${ }^{4}$, Valcinir A.S. \\ Vulcani $^{5}$, Samuel M.G. da Silva ${ }^{3}$ e Jair A. Ferreira Júnior ${ }^{6}$ \\ ABSTRACT.- Sant'Ana F.J.F., Oliveira S.L., Rabelo R.E., Vulcani V.A.S., Silva S.M.G. \& Ferreira \\ Júnior J.A. 2012. [Outbreaks of Piscinoodinium pillulare and Henneguya spp. infection \\ in intensively raised Piaractus mesopotamicus in Southwestern Goiás, Brazil.] Sur- \\ tos de infecção por Piscinoodinium pillulare e Henneguya spp. em pacus (Piaractus meso- \\ potamicus) criados intensivamente no Sudoeste de Goiás. Pesquisa Veterinária Brasileira \\ 32(2):121-125. Laboratório de Patologia Veterinária, Campus Jataí da Universidade Federal \\ de Goiás, Jataí, GO 75801-458, Brazil. E-mail: santanafjf@yahoo.com \\ Three outbreaks of infection by Piscinoodinium pillulare and Henneguya spp. in Piarac- \\ tus mesopotamicus intensively raised in Southwestern Goiás, Brazil, are described. Two ou- \\ tbreaks occurred in the winter and another one in summer. Morbidity (58.57\%-90\%) and \\ mortality $(100 \%)$ rates were determined. Stress episodes occurred previously in all cases. \\ Clinical signs included behavior changes and erratic movements with loss of equilibrium. \\ Main gross findings were swelling of the gills with white mucus exudate. Microscopically, \\ gills had mild to moderate multifocal necrotizing and lympho-plasmocytic inflammation \\ with hypertrophy and fusion of secondary lamellae. In addition, numerous intralesional \\ trophonts of P. pillulare and cysts of Henneguya spp. were noted. According to our know- \\ ledge, this seems to be the first description of Piscinoodinium pillulare e Henneguya spp. \\ infection in fishes reared in Southwestern Goiás, Brazil.
}

INDEX TERMS: Fish diseases, fish farm, Piscinoodinium pillulare, Henneguya spp.

RESUMO- Três surtos de infecção por Piscinoodinium pillulare e Henneguya spp. em Piaractus mesopotamicus criados intensivamente no Sudoeste de Goiás são descritos. Dois surtos ocorreram no inverno e um no verão. As taxas de morbidade $(58,57 \%-90 \%)$ e mortalidade $(100 \%)$ foram determinadas. Episódios de estresse ocorreram previamente em todos os casos. Sinais clínicos incluíam mudanças comportamentais e movimentos erráticos com perda de equilíbrio. Os principais achados macroscópicos foram tumefação das brânquias com exsudato mucoso esbranqui-

\footnotetext{
${ }^{1}$ Recebido em 1 de setembro de 2011.

Aceito para publicação em 10 de outubro de 2011.

${ }^{2}$ Laboratório de Patologia Veterinária (LPV), Universidade Federal de Goiás (UFG), Campus Jataí (CAJ), BR 364 Km 192, Jataí, G0 75801-458, Brasil. *Autor para correspondência: santanafjf@yahoo.com

${ }^{3}$ Laboratório de Bioquímica, UFG-CAJ, Jataí, GO.

${ }^{4}$ Setor de Cirurgia de Grandes Animais, UFG-CAJ, Jataí, GO.

${ }^{5}$ Laboratório de Anatomia, UFG-CAJ, Jataí, GO.

${ }^{6}$ Acadêmico de Medicina Veterinária, Laboratório de Patologia Veterinária, Universidade Federal de Goiás, Campus Jataí, BR 364 Km 192, Jataí, GO 75801-458. Bolsista PIBIC/CNPq.
}

çado. Microscopicamente, as brânquias apresentaram inflamação linfoplasmocítica e necrosante, multifocal, leve a moderada, com hipertrofia e fusão de lamelas secundárias. Adicionalmente, numerosos trofontes intralesionais de $P$. pillulare e cistos de Henneguya spp. foram notados. Para o conhecimento dos autores, essa parece ser a primeira descrição de infecção por P. pillulare e Henneguya spp. em peixes criados no Sudoeste de Goiás.

TERMOS DE INDEXAÇÃO: Doenças de peixes, piscicultura, Piscinoodinium pillulare, Henneguya spp.

\section{INTRODUÇÃo}

A piscicultura tem se destacado nos últimos anos por representar importante e saudável fonte protéica para a alimentação humana. Em função das características territoriais, hídricas e climáticas favoráveis, a atividade vem se expandindo no território brasileiro com resultados promissores, principalmente em criações intensivas com espécies de água doce (Pavanelli et al. 2002). Entretanto, caso não sejam tomadas medidas profiláticas e de manejo sanitário 
adequadas, pode ocorrer à introdução de diversos patógenos e enfermidades que podem acarretar prejuízos consideráveis e, em alguns casos, inviabilizar o sistema produtivo. Doenças parasitárias na piscicultura representam as causas mais comuns e importantes de prejuízos em criações de cativeiro (Thatcher \& Brites-Neto 1994). Dentre essas enfermidades, numerosas infecções causadas por protozoários e metazoários destacam-se pelos altos índices de mortalidade (Pavanelli et al. 2002).

Piscinoodinium pillulare (Schäperclaus 1954) Lom 1981 é um protozoário mastigóforo, dinoflagelado, altamente patogênico, que afeta as brânquias e a superfície corporal de peixes criados em regiões tropicais e temperadas. Foi descrito no Brasil pela primeira vez em 2001, no Estado de São Paulo (Martins et al. 2001). Os peixes afetados geralmente apresentam sinais de desconforto e de asfixia, movimentos operculares intensos e dobras nas nadadeiras. Em alguns casos, ocorre uma camada superficial cutânea de aspecto aveludado. Por essa razão, a infecção é conhecida popularmente como "doença do veludo". As principais alterações consistem de petéquias, necrose e inflamação no tegumento, além de hipertrofia e fusão extensa de lamelas secundárias. Essas últimas lesões são consideradas mais graves, pois resultam em grave dificuldade respiratória e consequente morte dos animais (Afip 1997/1998, Pavanelli et al. 2002).

0 gênero Henneguya Thélohan 1892 (Myxozoa: Myxobolidae) está entre os metazoários mixosporídeos mais abundantes na América do Sul, compreendendo 40 espécies identificadas no Brasil (Eiras et al. 2010). Henneguya spp., assim como P. pillulare, também causa infecções cutâneas e branquiais em peixes de águas quentes (Mitchell 1978), resultando em mortalidades expressivas e impacto econômico considerável (Martins et al. 1997, Adriano et al. 2005). Os sinais clínicos e as lesões observadas em peixes afetados por esse metazoário são muito semelhantes aos evidenciados na infecção por P. pillulare (Martins et al. 1997).

Diante da falta de informações epidemiológicas, clínicas e patológicas de enfermidades de importância econômica em peixes na região central do Brasil, esse trabalho tem por objetivo descrever três surtos de infecção por $P$. pillulare e Henneguya spp. em Piaractus mesopotamicus (pacu) criado intensivamente no Sudoeste de Goiás.

\section{MATERIAL E MÉTODOS}

Foram observados três surtos de mortalidade em pacus criados intensivamente no município de Jataí (latitude -170 52' 53”, longitude -51을 42' 52", altitude $696 \mathrm{~m}$ ), sudoeste de Goiás, entre 2009 e 2011. Dois surtos ocorreram em propriedades comerciais de criação intensiva de piscicultura e outro surto foi diagnosticado em um criatório tipo "pesque-pague". De cada surto, três peixes recentemente mortos foram enviados para necropsia no Laboratório de Patologia Veterinária da Universidade Federal de Goiás, Campus Jataí. O histórico clínico e epidemiológico foi obtido com os proprietários e tratadores. Na necropsia, foram realizados raspados na superfície corporal e nas brânquias dos animais. 0 material obtido foi montado entre lâmina e lamínula com solução fisiológica e observado em microscópio óptico. Fragmentos de brânquias foram fixados em formalina 10\% tamponada, proces- sados rotineiramente para histopatologia, corados com hematoxilina-eosina (HE) para realização de cortes com cinco micrômetros de espessura.

\section{RESULTADOS}

O surto um ocorreu em dezembro de 2009 em um criatório tipo "pesque-pague". A temperatura na época oscilava de 18 a $31^{\circ} \mathrm{C}$. 0 proprietário havia recentemente comprado um lote de peixes com idade variando entre dois e três anos e pesando de 2 a $4 \mathrm{~kg}$. Os peixes foram transportados de outra propriedade e o trajeto rodoviário durou aproximadamente três horas. Segundo o proprietário, houve superlotação nos tanques de transporte. Após a chegada, os peixes foram colocados abruptamente em um tanque. Dois dias após a chegada, iniciaram os primeiros sinais clínicos da enfermidade e a mortalidade. De um total de 7.000 peixes recém-alojados, $4.100(58,57 \%)$ adoeceram e morreram.

0 surto dois foi observado em um criatório de piscicultura comercial no mês de junho de 2010, onde as temperaturas médias durante a madrugada e as manhãs eram de $9-19^{\circ} \mathrm{C}$. O problema surgiu em apenas um tanque que abrigava peixes de oito a doze meses de idade, que pesavam de 0,7 a $0,8 \mathrm{~kg}$. De um plantel de 2.000 peixes, 1.800 $(90 \%)$ adoeceram e morreram em um intervalo de 15 dias. Na propriedade, haviam outros dois tanques que criavam outras espécies de peixes, como Pseudoplatystoma corruscans (pintado) e Astyanax spp. (lambari), mas que não manifestaram nenhum problema clínico durante o surto.

0 surto três ocorreu em uma propriedade de piscicultura de criação intensiva, em maio de 2011. Na semana do ocorrido, em quatro dias consecutivos, as temperaturas noturnas e matinais oscilaram de $8-13^{\circ} \mathrm{C}$. De dois tanques que continham 500 peixes de sete meses de idade e $0,7 \mathrm{~kg}, 420$ (84\%) adoeceram e morreram.

Os sinais clínicos manifestados pelos animais nos três surtos foram semelhantes e consistiam de mudança de comportamento, apatia ou movimentos erráticos com perda de equilíbrio e isolamento na periferia dos tanques, especialmente próximos a superfície e a entrada de água do tanque. Alguns peixes afetados se aglomeravam nas margens dos tanques. A ingestão de ração diminuiu consideravelmente em todos os casos. No surto um, alguns peixes apresentavam endoftalmia. 0 curso clínico foi de poucas dias no surto um.

Macroscopicamente, observaram-se as brânquias tumefeitas e pálidas com conteúdo mucoso esbranquiçado (Fig.1). Esse exsudato também era encontrado, geralmente em menor quantidade, na superfície corporal dos peixes afetados. Nos surtos um e dois, a superfície corporal dorsal e a cabeça apresentavam um aspecto brilhante com uma fina camada de muco levemente esverdeado. Em alguns peixes do surto um, notou-se a cabeça com superfície seca, opaca e irregular.

No exame histológico das brânquias, foram notados branquite linfoplasmocítica necrosante multifocal leve a moderada com áreas multifocais de hipertrofia e fusão de lamelas secundárias. Associada a essas lesões foram detectados numerosos trofontes (protozoários) ovais ou piriformes, medindo $64-100 \mu \mathrm{m}$, que estavam geralmente aderidos ao epitélio, com 
morfologia consistente com Piscinoodinium pillulare (Fig.2 e 3). Alguns poucos cistos de $70-130 \mu \mathrm{m}$ de diâmetro possuíam esporos fusiformes e alongados, identificados como Henneguya spp. (Fig.3). Tanto P. pillulare, quanto Henneguya spp.

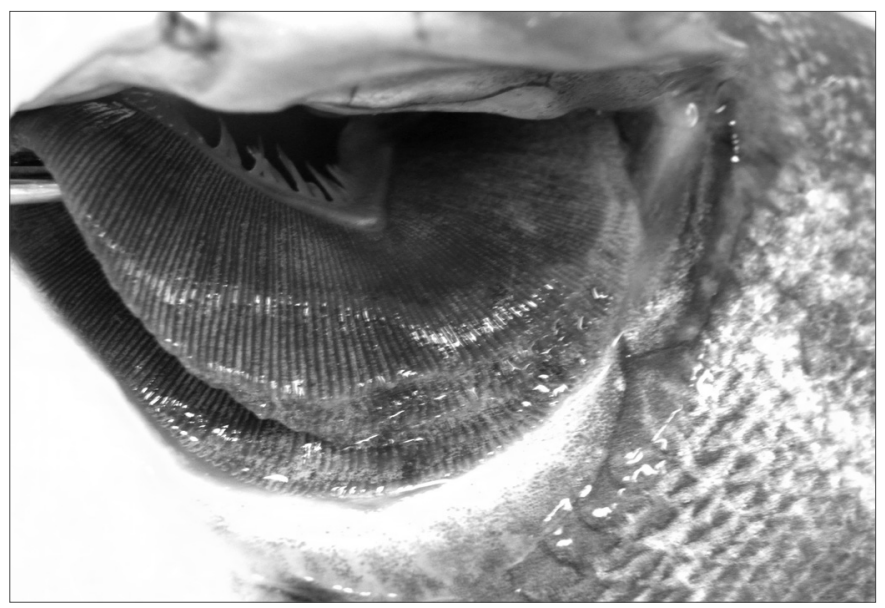

Fig.1. Piaractus mesopotamicus com áreas multifocais de palidez nas brânquias e acúmulo de exsudato mucoso esbranquiçado (Surto 1).

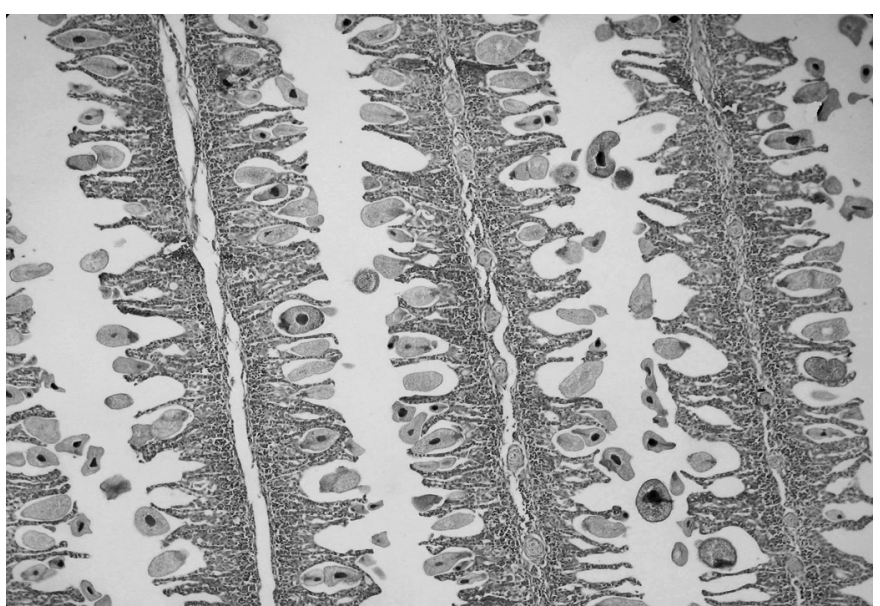

Fig.2. Brânquias com trofontes de Piscinoodinium pillulare aderidos ou não às lamelas secundárias. Observar hipertrofia, inflamação e fusão de lamelas (Surto 3). HE, barra = $175 \mu \mathrm{m}$.

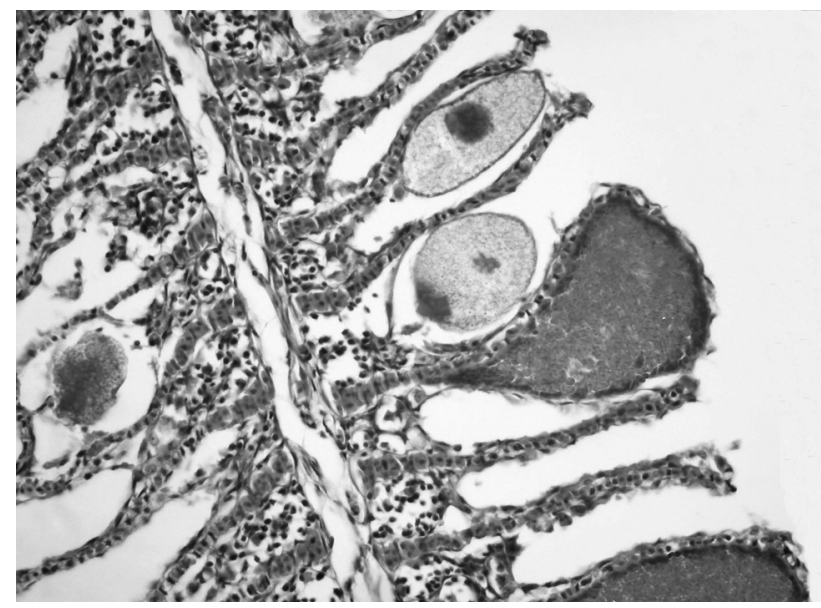

Fig.3. Brânquias com cistos de Henneguya spp. (setas pretas) e Piscinoodinium pillulare (setas brancas) (Surto 3). HE, barra $=84 \mu \mathrm{m}$. foram detectados nas regiões basais, intermediárias e apicais das lamelas afetadas. Na pele, não foram observadas alterações significativas; notou-se apenas raros trofontes de $P$. pillulare. No exame citológico do muco, $P$. pillulare e Henneguya spp. também foram evidenciados.

\section{DISCUSSÃO}

A criação de pacus, tanto em pisciculturas comerciais convencionais quanto em "pesque-pague", na Região Sudoeste de Goiás assume grande importância econômica local, em função do valor comercial do produto, da grande demanda regional, do bom desempenho reprodutivo e do potencial para aquicultura local. Todavia, erros de manejo como situações de estresse e maior concentração de peixes por tanques pode facilitar a ação de agentes patogênicos nos tanques, em comparação com ambientes naturais (Thatcher \& Brites-Neto 1994). Algumas enfermidades protozoárias de peixes ocorrem com frequência após práticas de manejo realizadas em épocas frias do ano ou associadas a outros fatores estressantes (Ceccarelli et al. 1990, Thatcher \& Brites-neto 1994, Martins et al. 2001, Moraes \& Martins 2004). No presente estudo, todos os surtos foram precedidos de situações graves de estresse que, provavelmente, desencadearam o desenvolvimento das lesões parasitárias. No surto um, o transporte, a alta densidade de estocagem e a liberação abrupta dos peixes no tanque e, nos surtos dois e três, as baixas temperaturas locais, foram consideradas cruciais na patogenia das infecções. Quadro muito semelhante foi observado em surtos de infecção por Piscinoodinium pillulare e Anacanthorus penilabiatus Boeger, Husak et Martins 1995 (Platyhelminthes: Monogenoidea) em pacus criados em pesque-pagues (Tavares-Dias et al. 2001a). Surtos com altos índices de mortalidade por infecção por $P$. pillulare são comuns em épocas frias do ano, porém, nesses casos não se registra aumento da quantidade de parasitas, e sim, diminuição da resistência imunológica dos peixes (Tavares-Dias et al. 2001a). Alguns autores afirmam que os mixosporídeos podem estar presentes naturalmente nos habitats ou em criações de cativeiros de peixes e que os sinais clínicos da enfermidade podem surgir quando houver desequilíbrio entre hospedeiro, parasita e meio ambiente (Lom \& Noble 1984). Além dos fatores acima mencionados, a qualidade baixa da água e o alto conteúdo de matéria orgânica podem influenciar na patogenia de $P$. pillulare (Martins et al. 2001, Piazza et al. 2006).

Não foi possível determinar a forma exata de introdução dos protozoários e metazoários nos criatórios descritos nesse estudo, porém os autores acreditam que nos surtos dois e três, os agentes já estavam nos tanques e/ou nos peixes e, após a condição estressante (baixas temperaturas), houve imunodepressão e exacerbação dos efeitos patológicos dos parasitas. Adicionalmente, no surto um, os peixes transportados podem ter carreado os agentes para o tanque. É comum, na transferência de peixes de pisciculturas de origem para pesque-pagues, a disseminação de agentes com potencial patogênico (Martins et al. 2000). Alguns autores afirmam que animais selvagens, como aves piscívoras atraídas para os tanques de cultivo, podem transmitir infecções entre os tanques, principalmente veiculando esporos de mixosporídeos (Thatcher 1991). 
Infecções parasitárias por P. pillulare e Henneguya spp. já foram diagnosticadas em outras espécies de peixes no Brasil, como Prochilodus lineatus (curimbatá) (Campos et al. 2011), Leporinus macrocephalus (piauçú) (Martins et al. 1999, Tavares-Dias et al. 2001a,b), Pseudoplatystoma corruscans (pintado) (Eiras et al. 2009), Cyphocharax nagelli (saguiru) (Martins \& Onaka 2006), Cyprinus carpio (carpa) (Schalch \& Moraes 2005), Oreochromis niloticus (tilápia) (Martins et al. 2001), Colossoma macropomum (tambaqui) (Schalch \& Moraes 2005) e híbrido tambacu (cruzamento de fêmea de C. macropomum com macho de P. mesopotamicus) (Martins et al. 2002). P. pillulare também já foi detectado parasitando as brânquias de peixes ornamentais amazônicos, como Carnegiella martae (peixe borboleta) (Tavares-Dias et al. 2010). Esses parasitas já foram identificados infectando peixes criados em pisciculturas comerciais (Martins et al. 1999, 2001, 2002, Naldoni et al. 2009), mantidos em "pesque-pague" (Tavares-Dias et al. 2001a,b, Schalch \& Moraes 2005, Schalch et al. 2006) ou em rios brasileiros (Martins \& Onaka 2006, Eiras et al. 2009, Tavares-Dias et al. 2010, Campos et al. 2011). Essas infecções já foram registradas em várias localidades brasileiras, como a região amazônica (Tavares-Dias et al. 2010) e os estados de São Paulo (Martins et al. 1997, 1999, 2001, Tavares-Dias et al. 2001a,b, Schalch \& Moraes 2005, Martins \& Onaka 2006), Paraná (Eiras et al. 2009), Mato Grosso do Sul (Naldoni et al. 2009, Campos et al. 2011) e Santa Catarina (Piazza et al. 2006). Entretanto, registros dessas parasitoses não têm sido realizados em peixes criados no Estado de Goiás.

No presente surto, os sinais clínicos e as lesões detectadas nas avaliações macro e microscópica das infecções por P. pillulare e Henneguya spp. foram semelhantes às observadas em outras investigações (Martins et al. 1997, 1999, Afip 1997/1998, Schalch et al. 2006, Eiras et al. 2009, Naldoni et al. 2009). Em casos de infecção única por Henneguya spp., pontos brancos nas brânquias e nefrite crônica também podem ser observadas (Lima et al. 1995). Em algumas infecções mistas por P. pillulare e Henneguya spp. em peixes, podem não ocorrer alterações macroscópicas (Campos et al. 2011). No presente estudo, a morte dos peixes foi atribuída à insuficiência respiratória, devido ao comprometimento grave das brânquias e a procura dos peixes afetados por fontes de oxigênio nos tanques. As brânquias são estruturas que exercem função respiratória e mantém o equilíbrio iônico e osmótico dos peixes e sua integridade é essencial para a manutenção dessas funções (Oslon 1991).

Segundo a literatura compilada, são descritos pela primeira vez, os aspectos clínicos e patológicos das infecções por P. pillulare e Henneguya spp. em pacus criados comercialmente no Sudoeste de Goiás. Medidas profiláticas, de manejo e o regular acompanhamento da saúde dos animais precisam ser difundidos entre produtores para evitar perdas econômicas por enfermidades nos criatórios locais.

\section{REFERÊNCIAS}

Adriano E.A., Arana S. \& Cordeiro N.S. 2005. An ultrastructural and histopathological study of Henneguya pellucida n.sp. (Myxosporea: Myxobo- lidae) infecting Piaractus mesopotamicus (Characidae) cultivated in Brazil. Parasite 12:221-227.

AFIP 1997/1998. Case II. 15 ${ }^{\text {th }}$ Wednesday Slide Conference, Armed Forces Institute of Pathology. Disponível em http://vp4.afip.org/wsc/ wsc 97/97wsc15.htm

Campos C.M., Moraes J.R.E. \& Moraes F.R. 2011. Histopathology of gills of Piaractus mesopotamicus (Holmberg, 1887) and Prochilodus lineatus (Valenciennes, 1836) infested by monogenean and myxosporea, caught in Aquidauana River, State of Mato Grosso do Sul, Brazil. Revta Bras. Parasitol. Vet. 20:67-70.

Ceccarelli P.S., Figueira L.B., Lima C.L.B.F. \& Oliveira C.A. 1990. Observações sobre a ocorrência de parasitos no CEPTA entre 1983 e 1990. Bolm Téc. CEPTA 3:43-55.

Eiras J.C., Takemoto R.M. \& Pavanelli G.C. 2009. Henneguya corruscans n. sp. (Myxozoa, Myxosporea, Myxobolidae), a parasite of Pseudoplatystoma corruscans (Osteichthyes, Pimelodidae) from the Paraná River, Brazil: a morphological and morphometric study. Vet. Parasitol. 159:154158.

Eiras J.C., Takemoto R.M. \& Pavanelli G.C. 2010. Diversidade dos parasitas de peixes de água doce do Brasil. Clichetec, Maringá. 333p.

Lima C.L.B.F., Reis N.S., Cecarelli P.S. \& Bozano G.L.N. 1995. Modificações histológicas associadas com infecção por Henneguya sp. (Protozoa, Myxosporea) em pacu Piaractus mesopotamicus Holmberg, 1887 (Teleostei, Serrasalminae). Bolm Téc. CEPTA 8:13-23.

Lom J. \& Noble E.R. 1984. Revised classification of the class Myxosporea Bütschli, 1981. Folia Parasitologica 31:193-205.

Martins M.L., Souza V.N., Moraes F.R., Moraes J.R.E., Costa A.J. \& Rocha U.F. 1997. Pathology and behavioral effects associated with Henneguya sp. (Myxozoa: Myxobolidae) infections of captive pacu Piaractus mesopotamicus in Brazil. J. World Aquac. Soc. 28:297-300.

Martins M.L., Souza V.N., Moraes J.R.E. \& Moraes F.R. 1999. Gill infection of Leporinus macrocephalus Garavello \& Britski, 1988 (Osteichthyes: Anostomidae) by Henneguya leporinicola n.sp. (Myxozoa: Myxobolidae). Description, histopathology and treatment. Revta Bras. Biol. 59:527-534.

Martins M.L., Moraes J.R.E., Fujimoto R.Y., Onaka E.M., Nomura D.T., Silva C.A.H. \& Schalch S.H.C. 2000. Parasitic infections in cultivated brazilian freshwater fishes. A survey of diagnosticated cases. Revta Bras. Parasitol. Vet. 9:23-28.

Martins M.L., Moraes J.R.E., Andrade P.M., Schalch S.H.C. \& Moraes F.R. 2001. Piscinoodinium pillulare (Schäperclaus, 1954) Lom, 1981 (Dinoflagellida) infection in cultivated freshwater fish from the northeast region of São Paulo State, Brazil: parasitological and pathological aspects. Braz. J. Biol. 61:639-644.

Martins M.L., Onaka E.M., Moraes F.R., Bozzo F.R., Paiva A.M.F.C. \& Gonçalves A. 2002. Recent studies on parasitic infections of freshwater cultivated fish in the state of São Paulo, Brazil. Acta Scientiarum 24:981985.

Martins M.L. \& Onaka E.M. 2006. Henneguya garavelli n. sp. and Myxobolus peculiaris n. sp. (Myxozoa: Myxobolidae) in the gills of Cyphocharax nagelli (Osteichthyes, Curimatidae) from Rio do Peixe Reservoir, São José do Rio Pardo, São Paulo, Brazil. Vet. Parasitol. 137:253-261.

Mitchell L.G. 1978. Myxosporidan infections in some fishes of Iowa. J. Protozool. 25:100-105.

Moraes F.R. \& Martins M.L. 2004. Condições pré-disponentes e principais enfermidades de teleósteos em piscicultura intensiva, p.343-386. In: Cyrino J.E.P., Urbinati E.C., Fracalossi D.M. \& Castangnolli N. (Eds), Tópicos Especiais em Piscicultura de Água Doce Tropical Intensiva. TecArt, São Paulo.

Naldoni J., Arana S., Maia A.A.M., Ceccarelli P.S., Tavares L.E.R., Borges F.A., Pozo C.F. \& Adriano E.A. 2009. Henneguya pseudoplatystoma n. sp. causing reduction in epithelial area of gills in the farmed pintado, a South American catfish: histopathology and ultrastructure. Vet. Parasitol. 166:52-59.

Oslon K.R. 1991. Vasculature of the fish gill: anatomical correlates of physiological functions. J. Electron Microscopy Technique 19:389-405. 
Pavanelli G.C., Eiras J.C. \& Takemoto R.M. 2002. Doenças de peixes; profilaxia, diagnóstico e tratamento. 2a ed. EDUEM, Maringá. 305p.

Piazza R.S., Martins M.T., Guiraldelli L. \& Yamashita M.M. 2006. Parasitic diseases of freshwater ornamental fishes commercialized in Florianópolis, Santa Catarina, Brazil. Bolm Inst. Pesca 32:51-57.

Schalch S.H.C. \& Moraes F.R. 2005. Distribuição sazonal de parasitos branquiais em diferentes espécies de peixes em pesque-pague do município de Guariba-SP, Brasil. Revta Bras. Parasitol. Vet. 14:141-146.

Schalch S.H.C., Moraes F.R. \& Moraes J.R.E. 2006. Efeitos do parasitismo sobre a estrutura branquial de Leporinus macrocephalus Garavello e Britsk, 1988 (Anastomidae) e Piaractus mesopotamicus Holmberg, 1887 (Osteichthyes, Characidae). Revta Bras. Parasitol. Vet. 15:110115.
Tavares-Dias M., Martins M.L. \& Moraes F.R. 2001a. Fauna parasitária de peixes oriundos de "pesque-pague" do município de Franca, São Paulo, Brasil. I. Protozoários. Revta Bras. Zool. 18 (Supl.1):67-79.

Tavares-Dias M., Moraes F.R., Martins M.L. \& Kronka S.N. 2001b. Fauna parasitária de peixes oriundos de "pesque-pagues" do município de Franca, São Paulo, Brasil. II. Metazoários. Revta Bras. Zool. 18 (Supl.1):81-95.

Tavares-Dias M., Lemos J.R.G. \& Martins M.L. 2010. Parasitic fauna of eight species of ornamental freshwater fish species from the middle Negro River in the Brazilian Amazon Region. Revta Bras. Parasitol. Vet. 19:103-107.

Thatcher V.E. 1991. Amazon fish parasites. Amazoniana 11:263-572.

Thatcher V.E. \& Brites-Neto J. 1994. Diagnóstico, prevenção e tratamento das enfermidades de peixes neotropicais de água doce. Revta Bras. Med. Vet. 16:111-128. 